\title{
1 Regional Variation in British English Voice Quality
}

2

\author{
Erica Gold ${ }^{1}$, Christin Kirchhübel ${ }^{2}$, Kate Earnshaw ${ }^{1,3}$, and Sula Ross ${ }^{1}$ \\ University of Huddersfield ${ }^{1}$, Soundscape Voice Evidence ${ }^{2}$, J P French Associates ${ }^{3}$
}

\begin{abstract}
:
This study considers regional variation of voice quality in two varieties of British English Southern Standard British English and West Yorkshire English. A comparison of voice quality profiles for three closely related but not identical northern varieties within West Yorkshire is also considered. Our findings do not contradict the small subset of previous research which explored regional and/or social variation in voice quality in British English insofar as 'regionality' may play a small role in a speaker's voice quality profile. However, factors such as social standing and identity could perhaps be even more relevant. Even when considering homogeneous groups of speakers, it is not the case that there is a cohesive voice quality profile that can be attached to every speaker within the group. The reason for this, we argue, is the speaker-specificity inherent in voice quality.
\end{abstract}

\section{KEY WORDS: voice quality, vocal profile analysis, regional variation, British English, West Yorkshire English}

\section{Introduction}

It is acknowledged that voice quality (VQ) is a phonetic parameter that is likely to be subject to sociolinguistic and stylistic variation (Foulkes, 2002; Thomas, 2011). Despite this, voice quality has received less attention within the relevant research literature compared to other aspects of speech such as vowel and consonant realisations. While voice quality may be affected by regional/social background and speaking environment, it is also the case that voice quality is anatomically conditioned, insofar as it is related to the characteristics of a speaker's vocal apparatus, e.g. the length and thickness of the vocal folds, the habitual behaviour of the tongue, lips, jaw, larynx, and the anatomy of the nasal tract. It is for this reason that voice quality has gained ground within forensic speech science as it is a useful parameter for discriminating individual speakers. The primary focus of this study is to explore the trade-off between regionality and speaker-specificity of VQ by analysing different varieties of British English. Our analyses involve the VQ comparison between a northern and southern variety of British English, i.e. West Yorkshire (WY) and Standard Southern British English (SSBE), as well as between three closely related but not identical northern varieties, i.e. three localities within West Yorkshire. In addition to our primary aim, these comparisons will also allow us to comment on the potential relevance of social standing and identity on VQ. Finally, we take the opportunity to discuss the challenges surrounding perceptual VQ analysis and make suggestions for further developments that would aid socio-phonetic research (and forensic practice).

Voice quality is generally a "term used to describe a suprasegmental feature of speech that considers the habitual, anatomical settings an individual adopts when speaking. VQ is often described as capturing the characteristic sound of the voice, or the variations in the quality of what is being said over phrases or utterances rather than individual segments" (McIntyre et al., 2021: 281-282). VQ can be defined slightly differently in the literature depending on whether the researcher(s) consider only the phonatory settings or whether they take a more encompassing view of voice quality and consider supra-laryngeal features as well (see Esling et al. 2019 for a more in-depth overview of how VQ has been defined in the literature). In this investigation, voice quality follows the definitions set out by Abercrombie (1967) and Laver 
$(1980 ; 1994 ; 2000)$ whereby voice quality is used to refer to the long-term postures and settings of the entire vocal tract (i.e., from the vocal folds all the way up to any potential lip postures). Other researchers employing perceptual analysis of VQ have also taken this more encompassing definition (see for example, Stuart-Smith, 1999, Beck, 2005; Wormald, 2016, San Segundo et al., 2019).

\section{Literature review}

Voice Quality is analysed using a range of methods which fall within the following three primary approaches: acoustics, articulation, and perception. Acoustic VQ research will examine different spectral aspects of the speech signal, for example, considering amplitude differences between the first and second harmonics in order to evaluate creaky and breathy voice (Keating et al., 2015; Hillenbrand et al., 1994). Articulatory VQ research will be concerned with using equipment that allows researchers to investigate the movement, shape, and positioning of the tongue and lips, or the behaviour of the vocal folds, amongst others. For example, in order to examine tense, lax, breathy, and harsh voice in the Yi and Bai languages, Edmondson et al. (2001) use laryngoscopy to study the engagement of the laryngeal sphincter mechanism, larynx raising, movement of the epiglottal base, and the positioning of the apexes of the aryepiglottalic folds. Esling et al. (2019) provide an illustrative and detailed insight into a variety of instrumental techniques which have been employed to examine laryngeal behaviour. Perceptual VQ research is based on auditory analysis and will often involve a recognised protocol which allows the analyst to make judgements about laryngeal and/or supralaryngeal characteristics of the voice (e.g. Vocal Profile Analysis as described in Beck (2005), or the GRBAS protocol described in Hirano, 1981). Combined approaches to VQ analysis are also common whereby acoustic or articulatory analysis may be correlated with perceptual analysis, or articulatory analysis is correlated with acoustic analysis. An example is the work described in Keating et al. (2012), who investigated phonation in nine different languages by considering data from acoustic measurements ( $\mathrm{f0}$ and differences in harmonic amplitudes) as well as electroglottographic data. Another example is the work of Klug et al. (2019) which explores the possibility of using acoustic measurements in tandem with auditory assessments when making judgements about breathiness and creakiness in forensic speaker comparison casework.

Thomas (2011: 224) has stated that VQ is "uncharted territory in sociolinguistics" and that "language variationists generally regard it as a mysterious domain." In line with those claims, VQ research has historically been left largely to speech pathologists and clinicians. It is not surprising then that there are only very few studies which have examined VQ from a sociolinguistic perspective. The few studies that have explored VQ variation, have not always employed comparable methodologies in assessing VQ. Moreover, the sample sizes on which VQ observations were based are very small in many cases, and some of the research dates back to the late 1970s. These factors place limits on the generalisations that can be drawn for present day VQ variation.

Section 2.1 below summarises those studies that have focussed on variation in British English VQ and, collectively, these studies suggest that there may be evidence for social and regional variation in VQ - highlighting the value of further variationist research into this aspect of speech. This type of research would be of particular interest to the field of forensic phonetics, as experts, who carry out forensic phonetic casework, have identified VQ as potentially the most discriminant speech parameter in individuals (Gold and French, 2011). Therefore, there has been a growing body of VQ literature within the forensic phonetics community; however, the focus of these studies so far has been on methodological developments rather than on exploring variationist topics. 


\subsection{British English voice quality}

The majority of the studies focusing on VQ in the UK have provided regional descriptions based on auditory analysis. Knowles (1978) provides a detailed description of the articulatory settings that are characteristic of the Liverpool accent, termed the "Scouse voice". It is argued that the most interesting settings of Scouse are those of the velo-pharyngeal mechanism and the jaw. The velo-pharyngeal mechanism involves a group of organs acting together as a unit, whereby the tongue is retracted and raised, the pillars of the fauces are narrowed, the pharynx is tightened, the larynx is raised and the lower jaw is held close to the upper jaw (1978: 89). The primary auditory consequence of these settings is the 'adenoidal' quality of Scouse. A secondary effect is that all consonants are velarised, resulting in a 'dark' $/ 1 /$ in all positions (including those where Received Pronunciation has the 'clear' allophone) and tongue-tip consonants being produced instead with the blade (1978: 89).

Voice quality research on SSBE has been carried out by Stevens and French (2012) on 100 speakers from the Dynamic Variability in Speech (DyViS) database (Nolan et al., 2009). Stevens and French found that the majority of SSBE speakers shared a number of VQ settings, such as fronted tongue body, advanced tongue tip/blade, sibilance, breathy voice, and creaky voice (Stevens and French, 2012). San Segundo et al. (2019) carried out VQ analysis on the same set of speakers and their findings are in line with those of Stevens and French (2012). In addition, they also observe nasality and non-neutral vocal tract tenseness as common VQ settings amongst the speakers.

Wilhelm (2016) interviewed 30 participants, aged nine to over 50 years old, from North West Yorkshire. This area is defined as "Leeds, a mainly rural area comprising the former West Riding county, and a small area a few miles north of the Yorkshire Dales" (Wilhelm, 2016:1). Wilhelm found that the whole vowel system seemed to be compressed downwards in this region and therefore the accents were said to be characterised by a long-term lowering of the tongue-body (2016). As well as tongue body lowering, creaky voice was also said to be characteristic of speakers of North West Yorkshire English, with 29 out of the 30 participants perceived to "use a significant amount of creaky voice" (Wilhelm 2018:6). It was further suggested that permanent or intermittent use of velarized voice seemed to be an innovative feature favoured by teenagers (Wilhelm, 2016).

In his doctoral dissertation, Esling (1978) reports that voice quality settings were correlated with social class. Esling analysed 32 male speakers from two different wards in Edinburgh. His participants were divided into three different socio-economic classes. Results suggested that phonatory settings were most influenced by social class, with creaky voice appearing more in the highest of the three socio-economic groups, and harsh voice (and to a lesser extent whispery voice) being associated with the lowest of the three socio-economic groups. Similar social stratification was observed for supra-laryngeal and muscular tension settings; whereas non-neutral lip, jaw, and tongue positions as well as muscular tension features were present in the speech of the lowest socio-economic group, non-neutrality in these components was not characteristic of the higher socio-economic group.

Further to the Edinburgh study by Esling, Stuart-Smith (1999) also considered VQ in Scotland, but this time in Glasgow. Like Esling, Stuart-Smith, who analysed 32 male and female speakers (16 working class and 16 middle class) reports that VQ was correlated with class. Working class Glaswegian VQ was characterised by open jaw, raised and backed tongue body with possible tongue root retraction, and whispery voice. The middle-class Glaswegian speakers were best described as having the absence of the working-class VQ features (1999: 215). Stuart-Smith also uncovers correlations between VQ and age and gender; the 16 younger speakers had laxer supra-laryngeal articulations than the 16 older speakers. Males exhibited 
greater nasalization than female speakers, and differences in the males and females was also seen in the phonatory settings with males tending towards creaky voice, while females tended towards whispery voice. Unlike Knowles (1978) who reports a 'stereotypical' Scouse voice, Stuart-Smith presents little evidence for a stereotypical 'Glasgow voice'; however, speakers who were matched with respect to age, gender, and class showed similar VQ patterning.

Beck and Schaeffler (2015) carried out a perceptual VQ analysis of read speech from 76 speakers (31 male; 45 female; aged between 12-18 years) who were selected from the Voice of Young Scots (VOYS) database (Dickie et al., (2009). The participants were from three geographically distinct areas of Scotland; Inverness, Aberdeen, and Dumfries. The authors observed geographical differences for tongue tip/blade position, vertical tongue body position, and pharyngeal and laryngeal tension, but there were no significant phonation differences between the three geographically distinct regions. The authors suggest that the reason for the absence of phonation differences may be linked to the fact that the participants were adolescents. Beck and Schaeffler's findings corroborate those of Stuart-Smith (1999) with respect to VQ differences according to gender.

Wormald (2016) conducted an auditory analysis of VQ in Anglo English (AE) and Panjabi English (PE) men and women from Bradford and Leicester. The findings relating specifically to the AE males are considered to be the most relevant for present purposes. In terms of vocal tract features, the AE Bradford males were found to have neutral larynx height, non-neutral lingual postures, sibilance and non-neutral velopharyngeal settings (nasal and denasal) (2016: 129, 134). The AE Leicester males were found to have lingual fronting, sibilance, non-neutral velo-pharyngeal settings, and laryngeal tension as well as harsh, breathy, and creaky voice. It was noted that "among the AE males, Leicester males tend toward tension, and Bradford males towards laxness" (2016: 135).

Wormald's investigation also revealed how VQ varied according to language background, gender and age. For example, it was shown that "with respect to the PE speakers, larynx raising is the only setting consistently associated with these speakers and not the AE speakers." (2016: 135). With respect to age, it was demonstrated that "there is a difference between older and younger male speakers, with creaky voice increasing as age decreases, and breathiness increasing as age increases" (2016: 135).

Based on the literature presented here, the following observations can be drawn. In general, there does not appear to be regionally defined VQ settings or combinations of VQ settings. Knowles (1978) suggests the existence of a 'Scouse voice', but Stuart-Smith (1999) states that her findings do not support the concept of a 'Glaswegian' voice. Similarly, there is little evidence for the existence of a 'Yorkshire' voice, an 'SSBE' voice, or indeed a 'Bradford' or 'Leicester' voice. There are a number of VQ settings which occur in different regional varieties of English, e.g. both Glaswegian English and SSBE list 'advanced tongue tip' as a VQ feature. This would be evidence for the fact that VQ is not directly tied to the variety of English spoken but that other factors are involved including the speakers themselves. Having said this, the above studies do suggest that 'regionality' may have some relevance nevertheless; for example, whereas SSBE was shown to have a tendency for tongue body fronting, Scouse, and Glaswegian are said to feature tongue body retraction. In addition to 'regionality', age, gender, ethnicity, and social standing are also relevant.

Although the above studies based their assessment of VQ on auditory analysis, they are not methodologically homogeneous. Whilst some employ a recognised protocol in scoring VQ (e.g. Stuart-Smith (1999), Stevens and French (2012), Wormald (2016)), others appear to use a more holistic approach to VQ assessment (Knowles (1978) and Wilhelm (2016) - although Wilhelm also uses acoustic measurements, i.e. vowel formant area). The size and type of speaker samples used also differs between studies. Whilst Stevens and French analyse a large number of speakers who come from a very homogeneous group, Wilhelm appears to include 
rural as well as urban speakers in his sample. Wormald's study controls for gender, ethnicity, regional background, and age; as a result, the number of speakers who fall within a homogeneous group (e.g. young Anglo men from Bradford or Leicester) is 2; an extremely small sample. Out of the 8 studies listed above, 5 were conducted within the last 15 years, two were conducted in the late nineties/early noughties, and one dates all the way back to the late 1970 s.

There are only a small number of recent variationist studies exploring VQ. This, in addition to the methodological heterogeneity and small sample sizes, means that one has to be cautious in attempting to make generalisations with respect to regional VQ variation in British English. In the present study, we have addressed some of these methodological limitations by analysing sizeable groups of homogeneous speakers and applying a consistent methodological framework. Our analysis allows us to assess VQ variation across a macro-regional and microregional level. In turn, this will enable us to explore further the question of regionality and speaker specificity in VQ.

\section{Materials and methods}

\subsection{Materials}

The data for this study is drawn from two existing corpora: the West Yorkshire Regional English Database (hereafter WYRED; Gold et al., 2018) and the Dynamic Variability in Speech database (hereafter DyViS; Nolan et al., 2009). Both databases are publicly available ${ }^{1}$, and both were created with forensic speech science research in mind. WYRED includes 180 male speakers from West Yorkshire (Northern England), while DyViS includes 100 male speakers of Southern Standard British English (SSBE). Both databases include a 'Task 2' speaking task, which is directly comparable between the two databases as WYRED was modelled after DyViS. The data analysed in the present study comes from the studio quality version of Task 2 in both WYRED and DyViS. Task 2 consists of each participant speaking to a fictional accomplice (one of the research assistants) about the police interview they had just completed relating to a crime that they were involved in. The conversations are elicited over the telephone, but the participant (target speaker) is recorded at the near end of the telephone line resulting in studio quality recordings with $44.1 \mathrm{kHz}$ sampling rate and 16 bit resolution. The participants were also recorded over the telephone (resulting in telephone transmitted recordings) but these did not form part of the present analysis. The Task 2 conversations, which are around 15 minutes in length, elicited spontaneous, conversational speech for each participant.

Both databases contain rather homogenous populations insofar as they are all young, native British English males who grew up in English-speaking households and have no reported speech pathologies or hearing difficulties. Furthermore, all DyViS participants had been or were current students at the University of Cambridge, while WYRED contained mostly (but not all) students from the University of Huddersfield. Both databases only include male speakers, as the majority of forensic phonetic casework is carried out on male voices.

For the purposes of the research reported here, our analyses are based on 80 speakers in total: 60 speakers randomly-selected from WYRED (20 from Bradford, 20 from Kirklees, and 20 from Wakefield, see Figure 1 for map of local boroughs) and 20 speakers randomlyselected from DyViS (the yellow area within England on Figure 1 indicates the area of West Yorkshire relevant to WYRED; the black dot provides the location of Cambridge where the DyViS speakers were recorded). We accept that a 60 by 60 speaker design for SSBE and WY

\footnotetext{
${ }^{1}$ WYRED can be downloaded at https://reshare.ukdataservice.ac.uk/854354/.
} 
would have been a more ideal experimental set-up. However, the VQ findings reported by Stevens and French (2012) and San Segundo et al. (2019), where a total of 100 SSBE speakers were analysed, are very similar to our VQ findings. This offers reassurance that our findings, even though they are based only on a subset of 20 speakers, are nevertheless representative of the VQ tendencies established for SSBE. We therefore considered the value to be gained from an analysis of 40 further SSBE speakers to be disproportionate to the investment of time and costs involved in the additional analysis.

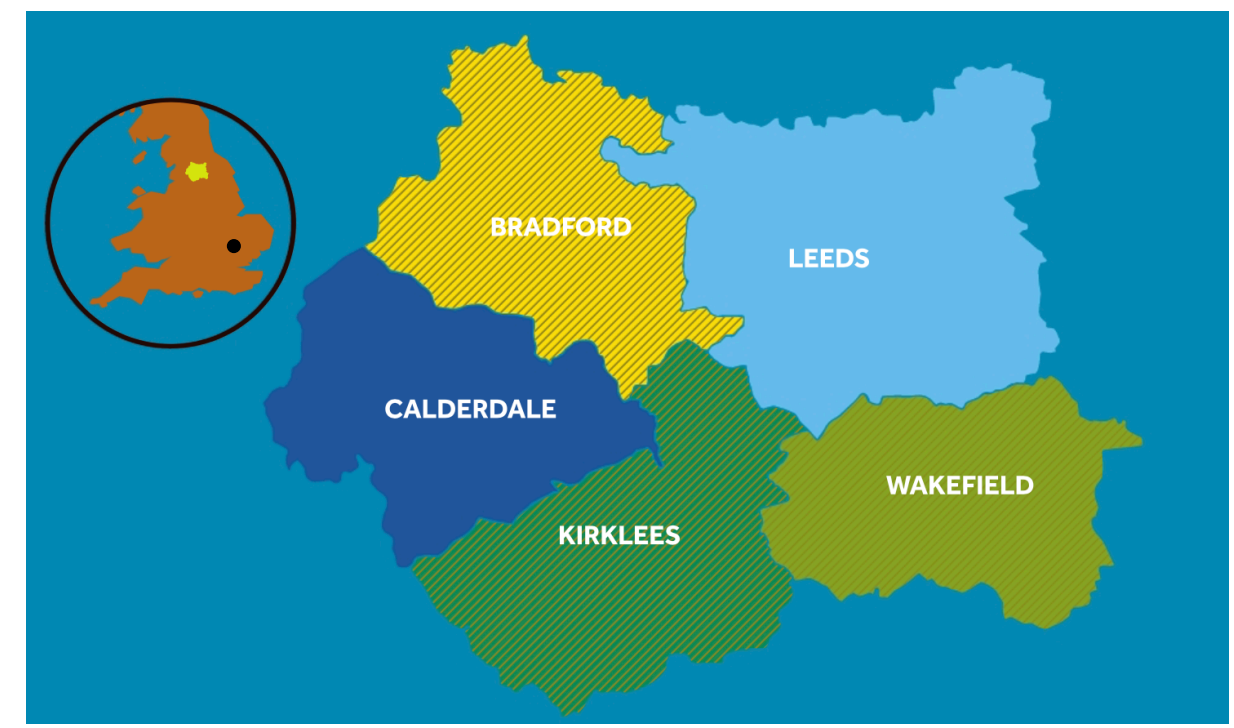

Figure 1: Map of West Yorkshire and the Local Boroughs

\subsection{Methods}

The voice quality analysis carried out in this paper follows closely the methodology employed by San Segundo et al. (2019). Specifically, this involved an auditory assessment of voice quality using the Vocal Profile Analysis (VPA) scheme (the reader is directed to Beck 2007 for a detailed introduction to the VPA). The 'original' VPA scheme had been designed for use in the clinical setting and included a number of prosodic, temporal and respiratory features in addition to a large stock of laryngeal and supra-laryngeal features. Whilst some of the features are rated in a binary way, i.e. present vs. absent, the majority of features are gradable into different degrees, and the original VPA protocol differentiates between six scalar degrees. The original scheme has been modified for the forensic setting by J P French Associates, and this modified version is the one used in the present study. The main differences between the 'original' and 'modified' protocols are a) the removal of prosodic, temporal, and respiratory settings, b) the merger of settings, e.g. fronted tongue body and raised tongue body were two separate settings in the 'original' protocol but were merged to 'fronted tongue body' in the 'modified' version, and c) the reduction from six scalar degrees to three scalar degrees, i.e. 'slight', 'marked', 'extreme'. For further detail regarding the differences between the 'original' and 'modified' versions, the reader is directed to San Segundo et al. (2019). 


\begin{tabular}{|c|c|c|c|c|c|c|c|c|}
\hline & \multicolumn{2}{|c|}{ FIRST PASS } & \multicolumn{5}{|c|}{ SECOND PASS } & \multirow[t]{3}{*}{ Notes } \\
\hline & \multirow{2}{*}{ Neutral } & \multirow{2}{*}{$\begin{array}{c}\begin{array}{c}\text { Non- } \\
\text { Neutral }\end{array} \\
\end{array}$} & \multirow{2}{*}{\multicolumn{2}{|c|}{ SETTING }} & Slight & Mark. & Extr. & \\
\hline & & & & & 1 & 2 & 3 & \\
\hline \multicolumn{9}{|c|}{ A. VOCAL TRACT FEATURES } \\
\hline \multirow[t]{5}{*}{ Labial } & & & \multicolumn{2}{|c|}{ Lip rounding/protrusion } & & & & \\
\hline & & & \multicolumn{2}{|l|}{ Lip spreading } & & & & \\
\hline & & & \multicolumn{2}{|c|}{\begin{tabular}{|l} 
Labiodentalisation \\
\end{tabular}} & & & & \\
\hline & & & \multicolumn{2}{|c|}{ Extensive labial range } & & & & \\
\hline & & & \multicolumn{2}{|c|}{\begin{tabular}{|l|} 
Minimised labial range \\
\end{tabular}} & & & & \\
\hline \multirow[t]{4}{*}{ Mandibular } & & & \multicolumn{2}{|l|}{ Close jaw } & & & & \\
\hline & & & \multicolumn{2}{|l|}{ Open jaw } & & & & \\
\hline & & & \multicolumn{2}{|c|}{ Extensive mandibular range } & & & & \\
\hline & & & \multirow{2}{*}{\multicolumn{2}{|c|}{\begin{tabular}{|l} 
Minimised mandibular range \\
Advanced tongue tip/blade
\end{tabular}}} & & & & \\
\hline \multirow[t]{4}{*}{ Lingual tip/blade } & & & & & & & & \\
\hline & & & \multicolumn{2}{|c|}{\begin{tabular}{|l|} 
Advanced tongue tip/blade \\
Retracted tongue tip/blade
\end{tabular}} & & & & \\
\hline & & & \multicolumn{2}{|l|}{ Retroflexion } & & & & \\
\hline & & & \begin{tabular}{|l|} 
Sibilance \\
\end{tabular} & & & & & \\
\hline Lingual body & & & Fronted tongu & & & & & \\
\hline & & & \begin{tabular}{|l} 
Backed tongu \\
\end{tabular} & & & & & \\
\hline & & & Extensive ling & range & & & & \\
\hline & & & \begin{tabular}{|l} 
Minimised lin \\
\end{tabular} & range & & & & \\
\hline Pharynx & & & Pharyngeal co & iction & & & & \\
\hline Velopharyngeal & & & Audible nasal & & & & & \\
\hline & & & Nasal & & & & & \\
\hline & & & \begin{tabular}{|l} 
Nasal w/o full \\
\end{tabular} & ease & & & & \\
\hline & & & \begin{tabular}{|l|} 
Denasal \\
\end{tabular} & & & & & \\
\hline Larynx height & & & Raised larynx & & & & & \\
\hline & & & $\begin{array}{l}\begin{array}{l}\text { Lowered laryn } \\
\text { expansion }\end{array} \\
\end{array}$ & haryngeal & & & & \\
\hline B. OVERALL MUSCULA & R TENSION & & & & & & & \\
\hline Vocal tract tension & & & Tense vocal tr & & & & & \\
\hline & & & Lax vocal trac & & & & & \\
\hline Laryngeal tension & & & Tense laryn $x$ & & & & & \\
\hline & & & Lax larynx & & & & & \\
\hline C. PHONATION FEATU & & & & & & & & \\
\hline & & & & & & Scalar De & & \\
\hline & & & & & Slight & Mark. & Extr. & \\
\hline & & & Neutral & Non-neutral & 1 & 2 & 3 & \\
\hline Voicing type & Voice & & & & & & & \\
\hline & Falsetto & & & & & & & \\
\hline & \begin{tabular}{|l|} 
Creak \\
\end{tabular} & & & & & & & \\
\hline & Creaky & & & & & & & \\
\hline Laryngeal frication & Whisper & & & & & & & \\
\hline & Whispery & & & & & & & \\
\hline & Breathy & & & & & & & \\
\hline & \begin{tabular}{|l|} 
Murmur \\
\end{tabular} & & & & & & & \\
\hline Laryngeal irregularity & Harsh & & & & & & & \\
\hline & Tremor & & & & & & & \\
\hline
\end{tabular}

\section{Figure 2: Modified Vocal Profile Scheme}

At the time of the study, three of the authors had had limited exposure to the VPA protocol. Therefore, an initial intensive VQ training session was provided by the second author who has had extensive exposure to the VPA protocol and frequently uses it to analyse VQ as part of forensic casework, research, and professional development. The training included a detailed coverage of the individual VPA settings and how they can be identified using auditory and acoustic information as well as proprioceptive techniques. As part of the training, the analysts completed practice ratings, the results of which were discussed within the group. On completion of the training, the analysts had reached general consensus on how VQ perceptions are mapped onto the VPA protocol.

Although all voices were initially rated by each of the four authors individually, the final VPA ratings are best described as group ratings rather than individual ratings. The purpose of this paper was not to assess inter-rater agreement in VPA analysis, even though it is acknowledged that this is an important topic. Indeed, inter-rater agreement in VPA analysis 
has been pursued in other studies (e.g. San Segundo et al., 2019, Kluge et al, 2018). Rather, the collaborative approach to VPA analysis employed in the current study reflects the practical forensic setting in which VPA ratings, arrived at as part of a speaker comparison analysis, are discussed amongst practitioners.

Two calibration sessions were used in arriving at the final VPA ratings. These sessions allowed the authors to check that there was consistency in their understanding of the individual settings. It allowed authors to calibrate their understanding of the scalar degrees, i.e. when is a voice 'slightly' creaky as opposed to 'markedly' or 'extremely' creaky, and it also provided space and time to raise and discuss challenging aspects of both VQ and VPA analysis.

Results were tabulated for each individual voice quality setting across each of the three

\section{Results}

The following section summarises the results of our VQ analysis. The reader will recall that we produced VPA profiles for 80 speakers of British English. Sixty speakers spoke with a West Yorkshire variety of English. We can further subdivide these 60 speakers according to locality within West Yorkshire with 20 speakers each from Bradford, Kirklees and Wakefield. The remaining 20 speakers spoke with Standard Southern British English. Each VPA profile involved the scoring of a range of different settings relating to phonation (laryngeal), vocal tract configurations (supra-laryngeal) and muscular tension. If a setting was present, the analyst had to assess whether the setting was present to a 'slight' degree (1), a 'marked' degree (2), or an 'extreme' degree (3). Section 4.1 considers the results from WY as a whole and SSBE, subsection 4.2 focuses on the three localities within WY, and finally 4.3 considers the connection between participants' evaluations of regional identity and VQ.

\subsection{Southern Standard British English and West Yorkshire English}

Figures 3 and 4 provide heat map visualisations of the VQ setting trends across the 20 SSBE speakers and the $60 \mathrm{WY}$ speakers. VQ settings are presented on the y-axis, while the scalar degrees are presented across the X-axis, the colour shading represents the proportion of speakers that exhibit a certain setting. If a box is very light in colour, fewer people exhibited this VQ setting, while those boxes that are very dark indicate that more speakers exhibit the particular VQ setting. 


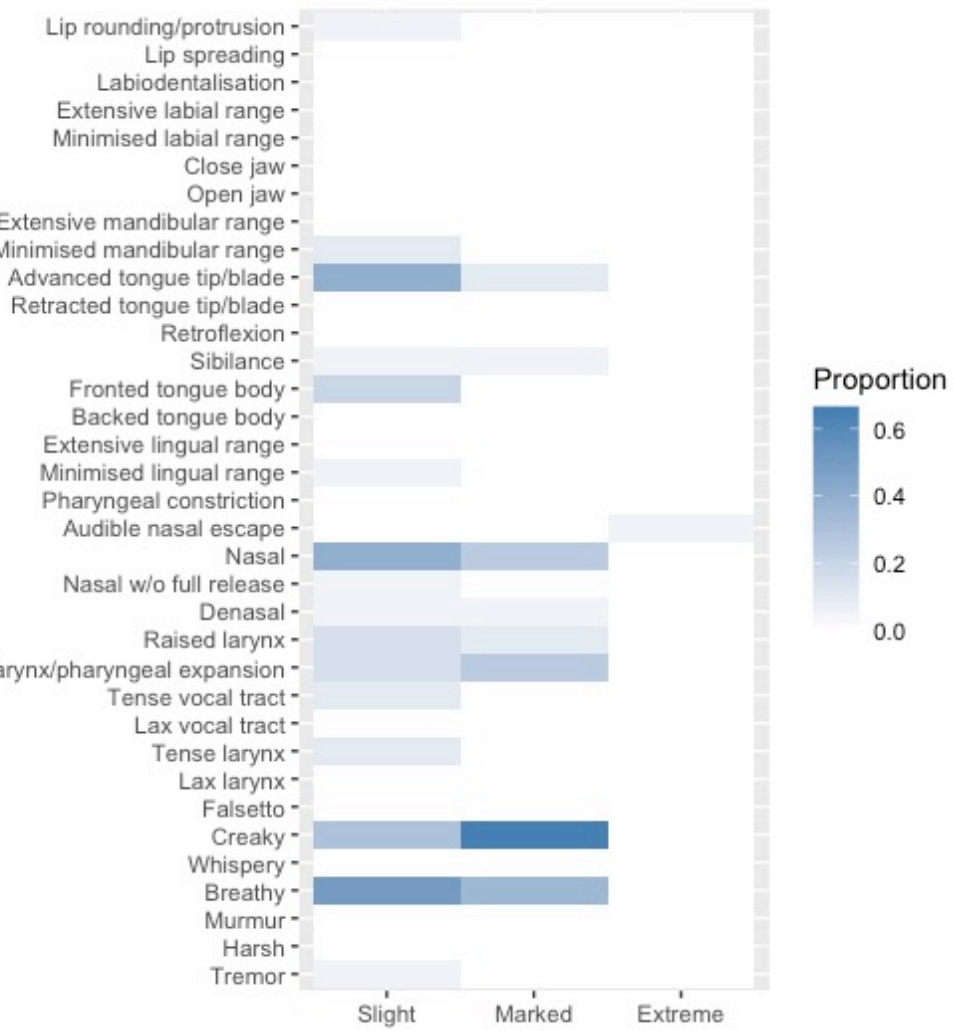

Figure 3: Scalar Degrees of VQ settings across SSBE

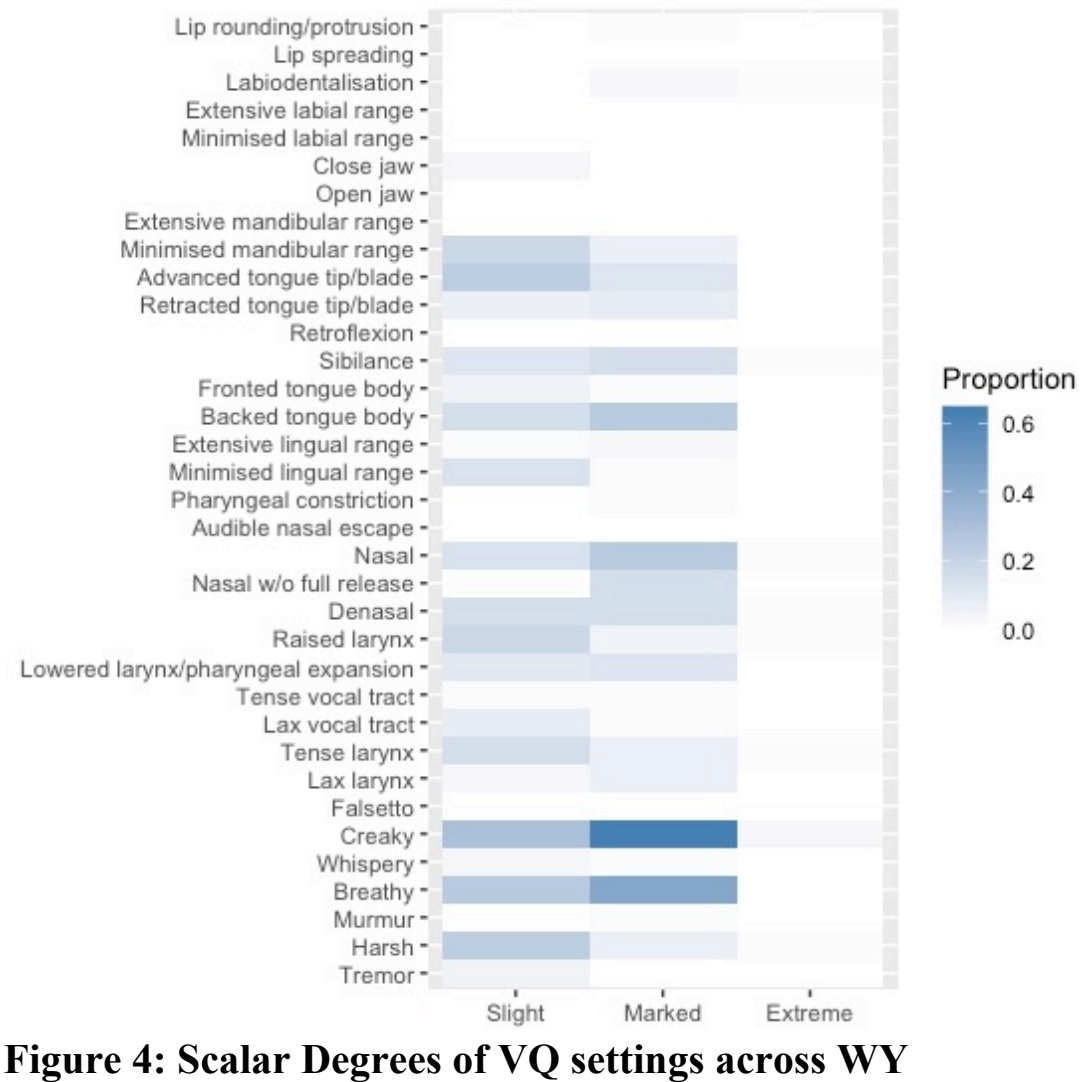

Lowered larynx/pharyngea

Lax vocal tract -

Laxe laryn

Creaky -

Breathy -

Harsh -

Slight Marked Extreme

339 The presentation of the data in Figures 3 and 4 provide clear visualizations of the most 340 prominent trends occurring in VQ settings across the two accent groups. Before commenting 
on the differences between SSBE and WY, it is of value to point out general VQ trends common to both groups of speakers.

The most striking individual VQ setting that both accent groups employ is creaky voice. a 'markedly' creaky voice. In addition to creaky voice there is a large proportion of speakers a 'markedly' creaky voice. In addition to creaky voice, there is a large proportion of speakers in both groups that also have breathy voice. Falsetto, murmur and tremor are rare (i.e. only 1 out of 80 speakers shows murmur, none display falsetto, and 3 have 'slight' tremor). In comparison to phonation, fewer speakers demonstrate non-neutrality with respect to supralaryngeal features in both accent groups. While there are speakers in both accent groups who show non-neutrality with respect to velo-pharyngeal settings, larynx height settings, and tongue body/tip settings, non-neutral muscular tension features are less common. Similarly, neither labial nor mandibular features occur very frequently in either SSBE or WY. It is rare that a speaker of either SSBE or WY demonstrates 'retroflexion', 'pharyngeal constriction', 'audible nasal escape', 'lip rounding/protrusion', 'lip spreading', 'extensive and minimal labial range', 'open jaw', 'extensive mandibular range'. Out of 80 speakers, the maximum number of speakers who displayed any of these settings is 1; some of these settings never occurred.

If there is non-neutrality in the supra-laryngeal settings, both accent groups show much more variability as compared to the phonatory settings, and it is not possible to identify a supralaryngeal setting which the majority of speakers, in either group, employ akin to 'creaky voice' for phonation. To exemplify, within both accent groups, there are speakers who have lax larynx and there are speakers who have tense larynx, there are speakers who have advanced tongue tip and there are speakers who have retracted tongue tip, there are speakers who have nasality and there are speakers who have denasality - all three pairs of these VQ settings are polar opposites. Not only is there variation in the direction of the VQ deviations, there is also variability in the types of settings speakers deviate. While some speakers deviate from neutral with respect to larynx height but show a neutral setting for tongue tip position (neither advanced nor retracted), for example, another speaker may have a neutral larynx (neither raised nor lowered) but an advanced tongue tip; again, this is the case for both accent groups.

With respect to scalar degree, very few SSBE and WY speakers show 'extreme' ratings for any of the 35 VPA settings considered. If non-neutrality occurs in the laryngeal, supralaryngeal, or muscular tension domains, this tends to be 'slight' or 'marked'. Whilst the absence of 'extreme' ratings would be of significance to the forensic practitioner, we would like to emphasise that it is possible that the absence of 'extreme' values is a result of the methodological design of the database, and therefore we place little weight on this aspect of the findings in the current paper.

In order to comment on the differences between SSBE and WY, we considered it helpful to present the findings in a slightly more general fashion. Rather than taking into account the scalar degrees as has been done in Figures 3 and 4 above, we tabulated the results on a presence/absence basis. As a result, settings that were evaluated as a 1 (Slight), 2 (Marked), or 3 (Extreme), are all counted equally here as being present and any VPA setting that does not appear in a speaker's profile is considered to be absent. Figure 5 provides a heat map visualisation of the VQ setting trends across the 20 SSBE speakers and the $60 \mathrm{WY}$ speakers using the presence/absence approach. VQ settings are presented on the y-axis, while the speaker group is presented on the x-axis. Again, the lighter the colour, the more speakers exhibited this setting. 


\section{Figure 5: Patterns in VQ settings across SSBE vs WY speakers}

Considering the differences between SSBE and WY, a large proportion of SSBE speakers have breathy and creaky phonation. Both types of phonation are also present in a large proportion of WY speakers. Harsh voice is an additional type of phonation which occurs amongst WY speakers but there are no SSBE speakers in the data that have harsh voice. Both SSBE and WY speakers show non-neutrality within the velo-pharyngeal setting but whilst the majority of SSBE speakers (who deviate from neutral) exhibit nasality, there is a split in the WY group with roughly an equal number of speakers demonstrating nasality and denasality ( $40 \%$ and $32 \%$ of speakers, respectively). A similar observation can be made for the tongue tip/blade. Both SSBE and WY demonstrate non-neutrality in this area but there is much less variability amongst SSBE speakers as compared to WY speakers with respect to the type of non-neutrality displayed. Whereas the former has a strong tendency for advanced tongue tip/blade, the latter demonstrate a mixture of advanced and retracted tongue tip/blade (WY has $35 \%$ of speakers with advanced tongue tip/blade and $15 \%$ of speakers with retracted tongue tip/blade, in comparison SSBE has $50 \%$ of speakers with advanced tongue tip/blade and $0 \%$ of speakers with retracted tongue tip/blade). Finally, whereas SSBE speakers have a tendency for a neutral tongue body setting, WY has a larger proportion of speakers with backed tongue bodies.

SSBE speakers appear to be much more homogenous in their VPA profiles compared to WY speakers - this is particularly true for the supra-laryngeal settings. This is apparent from the heatmaps which show that WY has more colours in the mid-range whereas SSBE has more colours in the extremes. One explanation for this could be the fact that the WY sample contained speakers from three different localities within Yorkshire. For this reason, we thought it worthwhile to look further into the different boroughs within WY to determine whether this variability diminishes when considering each of the three localities separately.

\subsection{Bradford, Kirklees, and Wakefield}


418 The VPA profile results for the 60 speakers in WY, separated into their boroughs, are presented below, SSBE is included for comparison purposes. Figure 6 provides an additional heat map of VQ settings like that presented in Figure 5.

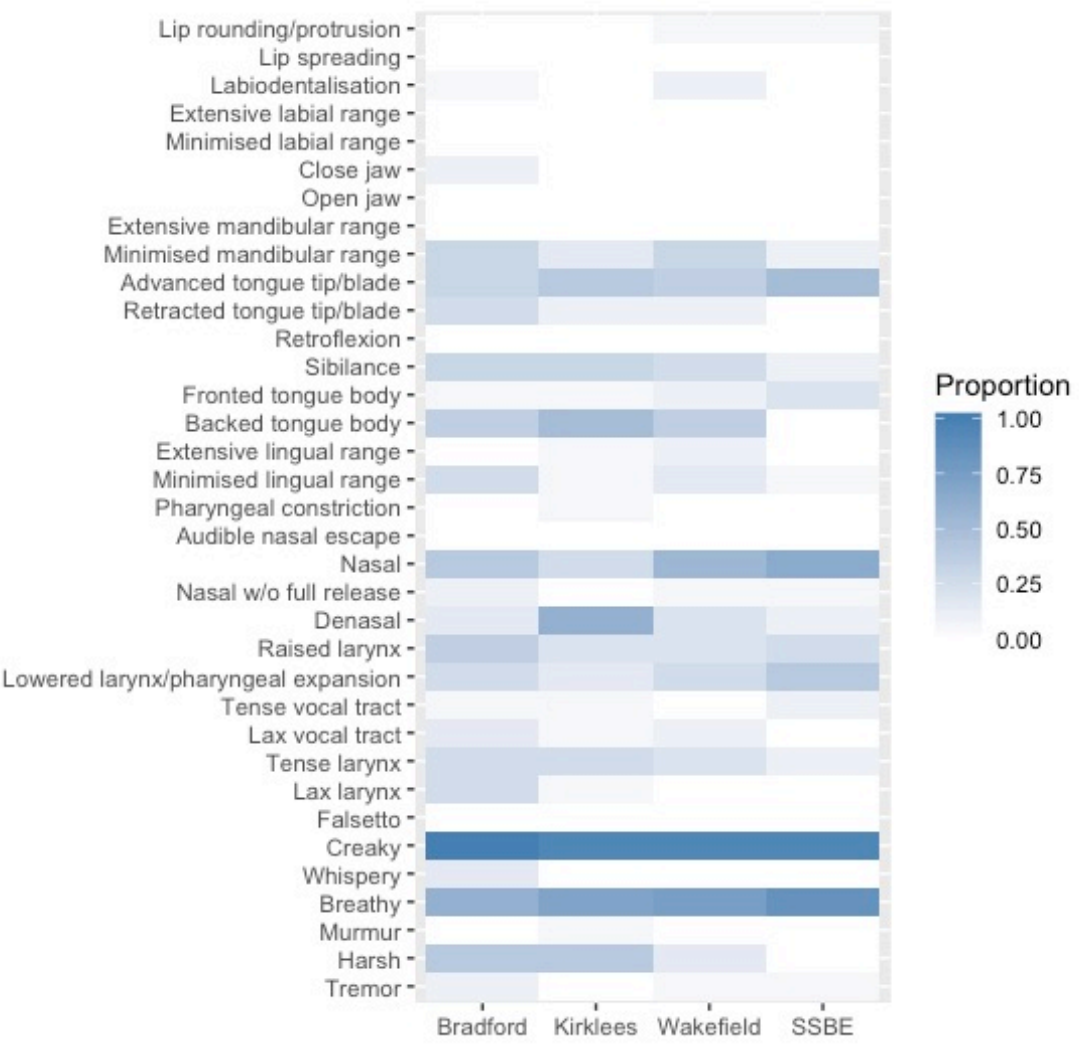

\section{Figure 6: Patterns in VQ settings within West Yorkshire}

The breakdown of the Bradford, Kirklees, and Wakefield data in Figure 6 exemplifies a similar level of variability across speakers as identified for the whole of WY above. Out of the three boroughs, Kirklees appears to be the most homogeneous but the level of homogeneity present within the SSBE sample is not replicated in either Bradford, Kirklees or Wakefield. A reason for this may be linked to differences in the degree of social cohesion and we will pick up on this again in the discussion below.

Many of the general VQ trends observed in section 4.1. above also hold true for the data here. Namely, non-neutral labial and mandibular features are much rarer in the populations of speakers compared to non-neutrality associated with tongue body and tip positions and those that are related to oral-nasal tract coordination, for example. Non-neutral phonation features are the most prevalent across the three boroughs. The most common non-neutral phonation across the three boroughs is creaky voice, followed by breathy and harsh voice. Bradford and Kirklees are more responsible for the tendencies towards harshness than Wakefield. Figure 6 confirms the finding established within the WY group, that there is variability within the velopharyngeal domain. Each of the three boroughs contain speakers who show nasality and speakers who show denasality. Kirklees is contributing most to the WY denasality results, while Bradford and Wakefield tend towards nasality, albeit Wakefield slightly more than Bradford. All three boroughs show a similar tendency toward backed tongue body and sibilance. Although all three boroughs have speakers with advanced and retracted tongue 445 


\subsection{Voice quality settings and self-evaluation of regional identity in West Yorkshire}

Previous research has shown that a speaker's identity, be it regional, social, occupational, ethnic, etc., is linked to their speech. For example, Hall-Lew et al. (2017) studied 12 months of political speeches (2011-2012) from 10 Scottish members of Parliament (MPs) and found that vowel height had significant correlation with political party affiliation. In her Middlesbrough data, Llamas (2007) found correlations between speakers' realisations of $/ \mathrm{p} \mathrm{t}$ $\mathrm{k} /$ and theirself-evaluated regional identities. Similarly, Devlin et al. (2019) observed that the nuclei of the MOUTH vowel in speakers from villages surrounding Sunderland were generally more raised amongst those speakers who affiliated strongly with Sunderland, where a raised nuclei $[\varepsilon v]$ is typically found. To our knowledge, the concept of regional identity has not yet been explored in relation to VQ and we are in a fortunate position in that the current WY data allows us to do just that, albeit only on a superficial level. As part of the data collection process for WYRED (but not for DyViS), participants were asked to self-evaluate their regional identity. All speakers were asked to fill in the blank for the following statement: "I most identify with being/being from

." Participants were then able to select from a closed list of: British, English, Yorkshire, West Yorkshire, Bradford, Huddersfield, Kirklees, or Wakefield. We explore the relationship between the self-evaluation of regional identity in WY and the most common VQ settings below.

Creaky voice and breathy voice were the most common VQ features in WY; however, they were consistently shared (and evenly distributed) across the three localities and therefore self-evaluated identity does not appear to play a role. The next five most common VQ features which were variable across the three localities were advanced tongue tip/blade, backed tongue body, nasality, denasality, and harsh voice. Out of these, only harsh voice emerged as a potentially interesting candidate and therefore we will only focus on this feature. Table 1 provides the proportion of participants that self-identified as a specific regional identity and whether they had a neutral or non-neutral (this includes slight, marked or extreme) VPA rating for harsh voice.

\begin{tabular}{|l|c|c|c|c|c|c|c|c|}
\hline \multicolumn{9}{|c|}{ Harsh Voice } \\
\hline & British & English & Yorkshire & $\begin{array}{c}\text { West } \\
\text { Yorkshire }\end{array}$ & Bradford & Huddersfield & Kirklees & Wakefield \\
\hline Number of Speakers & 19 & $8^{2}$ & 7 & 5 & 10 & 6 & 0 & 5 \\
\hline Non-Neutral & $16 \%$ & $13 \%$ & $43 \%$ & $60 \%$ & $50 \%$ & $83 \%$ & $0 \%$ & $20 \%$ \\
\hline Neutral & $84 \%$ & $88 \%$ & $57 \%$ & $40 \%$ & $50 \%$ & $17 \%$ & $0 \%$ & $80 \%$ \\
\hline
\end{tabular}

Table 1: Self-evaluation of regional identity against the presence or absence of harsh voice

There is evidence to suggest that speakers that evaluated themselves as having a national identity (i.e. British or English) rather than a northern regional one (i.e. Yorkshire, West Yorkshire, Bradford, Huddersfield, but not Wakefield) were much more likely to have no harshness in their voice. That is to say that those who self-evaluate with a northern regional identity title, rather than a national one, appear to be more likely to exhibit harsh voice. The suggestion that harshness may be linked to national vs. regional identity is further supported by a chi-square test, where British and English are combined as national identities, and Yorkshire, West Yorkshire, Bradford, Huddersfield, Kirklees, and Wakefield are combined as regional identities. Following these groupings, there is a chi-square statistic of 8.79 , with a $p$ value $=.003$, showing that national and regional groups of speakers use harshness significantly differently from one another.

\footnotetext{
${ }^{2}$ Figures equal $101 \%$ due to rounding.
} 


\section{Discussion}

Our results do not reveal a single Vocal Profile Analysis (VPA) setting that is tied to any of the regional accent varieties examined. It is not the case that Southern Standard British English (SSBE) invariably displays a VPA setting, or combination of settings, which is not present in West Yorkshire (WY) and vice versa. Having said this, a couple of observations, one from the laryngeal domain and one from the supra-laryngeal domain, warrant further discussion in this respect.

Firstly, WY speakers, as a whole, display a tendency for retracted tongue body. We would like to stress again that not every speaker out of the total of $60 \mathrm{WY}$ speakers showed non-neutrality in respect of tongue body position; however, if a speaker does display deviation from neutral then the tendency is for retraction rather than fronting. This is not the case for the SSBE speakers; indeed, if the tongue body was non-neutral for this accent group then the deviation generally results in fronting. This difference between WY and SSBE in tongue body setting tendencies links to the differences in segmental phonology between the two varieties. Although the WY and SSBE phonologies are very similar, they are not entirely uniform. For example, whereas WY speakers typically produce (very) dark /1/ tokens in syllable onset position (with dark /1/ involving tongue body retraction), SSBE speakers typically show a clear /1/ in that position (Wells, 1982: 370; see also Kirkham et al. 2020). Voice quality has had relatively little attention in the development of phonological theories; however, the 2019 work of Moisik et al. (also discussed in Esling et al. 2019) is ground-breaking insofar as it reconceptualises our understanding in this area. In particular, the work highlights the potential direct link between voice quality and vowel quality. Moisik et al. (2019) explore their VQ model in relation to phonologies of different languages. Although outside the scope of the current paper, it would be interesting to consider their model in relation to phonologies of different varieties of English in the UK and world-wide.

Secondly, harsh voice is a type of phonation which occurs in some of the WY speakers; none of the SSBE speakers feature harsh voice in comparison. The second author has extensive experience in analysing Yorkshire and South-East varieties of British English as part of forensic speaker comparison casework and the findings in relation to tongue body position corroborate those gained from her casework experience. In contrast, the finding that harsh voice is more prevalent in WY as opposed to South-East English is not something which has transpired in casework.

Whilst there may be slight regional tendencies in voice quality (VQ) on a macro-level, i.e. when comparing WY with SSBE, the data presented here does not suggest that there are micro-regional VQ differences, i.e. when comparing Bradford, Kirklees, and Wakefield. Given that the macro-regional comparisons only uncovered slight tendencies, this is entirely expected. The absence of consistent and reliable VQ differences between Bradford, Kirklees, and Wakefield is further supported by the fact that the authors would not be confident to assign a speaker to either of these localities purely taking into account VQ assessments.

The data revealed a difference between SSBE and each of Bradford, Kirklees, and Wakefield with respect to homogeneity of VQ. As indicated in section 3.1, care was taken that the speakers within each of the four groups were matched as far as possible with respect to age and language background. Furthermore, the speaking style elicited was comparable between the WY and SSBE speakers. Given the high level of comparability, it is surprising that the 20 SSBE speakers were a lot more similar to one another with respect to VQ compared to the 20 speakers of each of the three WY localities. A possible explanation for this may be the fact that SSBE is not merely a regional variety of English but also reflects a social dimension. Although all SSBE speakers were students at the University of Cambridge, it is unlikely that they all 
originated from Cambridge. It is fair to say that in terms of regional spread, the SSBE sample is probably more diverse compared to the WY group. However, unlike the WY group, the SSBE sample has a specific social profile, i.e. educated middle-class. The difference then in homogeneity in VQ between WY and SSBE may be an indication that social cohesion outweighs regional cohesion.

Although we only briefly touch on the concept of identity in relation to VQ, our data reveals that this too may be relevant. However, above and beyond geography, social class and self-evaluated identity, our data is persuasive in establishing the speaker-specific nature of VQ. Evidence for this can be seen in the fact that there is a large amount of variability within each of the accent groups. The variation is more striking in the WY sample as compared to the SSBE sample, but it is nevertheless present in the latter. Some VQ settings are more speaker-specific than others. Non-neutral tongue body and tip settings are more frequent than non-neutral labial or mandibular settings in all 80 speakers examined. Both creaky and breathy voice, in turn, are far more common than tongue body and tip settings. To exemplify, while it is rare for a WY or SSBE speaker to have 'lip rounding', it is common for a speaker of either accent to have a 'creaky voice'. Speaker-specificity, of course, does not just relate to single VQ settings but also to the combination of VQ settings characteristic for a particular speaker, i.e. the speaker's VQ profile. A VQ profile may include 'creaky voice' which is a frequent VQ setting; however, the VQ profile may also show deviations in other VQ settings resulting in a voice which is characterised by 'creaky voice in combination with tense larynx, and retracted tip'. Arguably, this VQ combination is far less common than either of the VQ settings in isolation. Indeed, this is how VPA findings are interpreted in forensic speaker comparison casework. It is not a case of how frequent is creaky voice, how frequent is tense larynx, etc. The question that the practitioner asks themselves is 'how common is this particular combination of VPA features?'.

The majority of the 80 speakers in our data have 'creaky voice' and a large proportion have 'breathy voice'. It is apparent from listening to the speakers that there are different types of 'creaky' and 'breathy' voice. Keating et al. (2015) have demonstrated how different types of creaky voice can be differentiated acoustically. We are not aware of a study that has thoroughly explored whether phoneticians are able to differentiate between different types of creaky voice, and if they could, how many types can be differentiated acoustically. Speaking purely from experience in analysing speech, the present authors can differentiate between a lax type of creaky voice and a tense type of creaky voice. The VPA currently does not allow for a distinction between these two types collapsing them into a single category of creaky voice. These types of perceptual nuances are not just the case for creaky voice but also for breathiness and the velo-pharyngeal domain. As with creaky voice, perceptually, there are different types of breathy voice and Ladefoged and Maddieson (1996), for example, distinguish between 'breathy voice' and 'slack voice'. Similarly, the interaction between the oral and nasal tract and the perceptual result of this is more complex than the three-way distinction offered by the VPA, i.e. nasality, denasality, and nasality without full release. Currently, the VPA does not have a way of capturing these nuances and therefore does not fully capture speaker-specificity in VQ. As a result, VPA analysis is generally used in conjunction with holistic analysis when assessing VQ in speaker comparison casework.

Difficulty in analysing VQ auditorily is often cited as the biggest obstacle in considering it in sociolinguistic and dialectology research (Thomas, 2011). In part, the difficulties stem from a lack of training and exposure to VQ analysis. Unlike auditory analysis of vowels and consonants, which is part of every undergraduate course in linguistics and which forms part of university assessments and competency tests (e.g., IPA Examination for the Certificate of Proficiency in the Phonetics of English), training in VQ analysis is confined to more specialist areas such as speech and language therapy and forensic speech science. It is 
not surprising that early studies showed low inter-rater agreement when analysing VQ (e.g. just above chance in Kreiman and Gerratt (1998).

The training given as part of the present work, undoubtedly, improved the authors' abilities, consistencies, and confidence in the auditory assessment of VQ; however, challenges in analysing VQ via the VPA protocol nevertheless remain. The challenges can be attributed to the complex and intricate settings that interact (or are inter-dependent) in order to give the overall VQ. They can also be attributed to the VPA protocol itself. Previous research has already discussed many of the challenges encountered with the VPA. For example, both Mackenzie Beck (2007) and San Segundo et al. (2019) highlight the presence of correlations between VPA settings, e.g. tense larynx correlating with raised larynx. San Segundo et al. (2019) also raise the point that some of the VPA settings are perceptually more salient than others and we agree with this observation.

Although we did not set out to test the reliability of VPA analyses, incidentally, our findings highlight that it is possible to achieve inter-rater agreement using VPA analysis. The 20 SSBE speakers that we analysed in this study were part of the 100 speakers previously analysed by Stevens and French (2012) and San Segundo et al (2019. Our findings are consistent with their results that many SSBE speakers demonstrate advanced tongue tip/blade, breathy voice, and creaky voice. We agree with the conclusion drawn in San Segundo et al. (2019), that high rates of inter-rater agreement can be achieved when using a clear methodological approach involving the VPA protocol.

Despite promising results on inter-rater agreements, we encourage further research that develops the VPA protocol. In order to do so, we should not shy away from exploring fundamental changes to our conceptualisation of VQ. The work of Esling et al. (2019) proposes such a fundamental change by the introduction of the Laryngeal Articulator Model (LAM). In this model the larynx is not just seen as a sound source but as a complex articulator similar to the tongue. We encourage research incorporating the ideas of the LAM model into the VPA protocol. Another avenue that could be explored in order to develop aspects of the VPA protocol is to introduce perceptual VQ categories, instead of mapping perception onto articulatory settings, as is currently the case. This would involve a change of terminology, e.g. rather than scoring a voice as demonstrating 'minimised range of lingual body movement' one would describe the voice as exhibiting 'articulatory imprecision'. As a result, analysis would focus more on the auditory perception of VQ rather than on the perceptual production of VQ. This may help alleviate certain limitations related to potential vocal profile setting interdependencies, further improve inter-rater agreement, and also allow variationist VQ research to focus on salient perceptual similarities and differences rather than estimating the mechanics of the voice quality we perceive.

\section{Conclusion}

Overall, our findings showed that almost all speakers in our sample have some degree of creaky voice, and a large proportion of speakers also have breathy voice. In comparison to phonation, fewer speakers demonstrate non-neutrality with respect to supra-laryngeal features in both WY and SSBE. Specifically, for phonation, harsh voice occurs amongst WY speakers, while our SSBE sample did not include any speakers with harsh voice. We also found that our data may support the notion that harsh voice in WY may carry some type of social identity marker insofar as those identifying with more regional affiliations (aside from those identifying as Wakefield) were more likely to produce harsh voice compared to their nationally identifying counterparts. And finally, in general, it was found that SSBE speakers appear much more homogenous in their overall VPA profiles compared with the WY speakers, which is especially true of the supra-laryngeal settings. 
Our findings do not contradict the observations that can be drawn from the small set of previous research which dealt with regional and/or social variation in VQ in British English. Whilst 'regionality' may play a small role in a speaker's VQ profile, factors such as social standing and identity could perhaps be even more relevant. However, even when considering homogeneous groups of speakers, it is not the case that there is a cohesive VQ profile that can be attached to every speaker within the group. The reason for this, we argue, is the speakerspecificity inherent in VQ. For the forensic practitioner - even though the data presented here covers very specific 'populations' of speakers, we believe it goes some way in filling a gap. We hope this paper provides a useful resource for casework, training, and future research.

For the sociolinguist/phonetician - we appreciate that the paper does not include the

\section{References}

Abercrombie, David. 1967. Elements of general phonetics. Edinburgh: Edinburgh University Press.

Beck, Janet. 2005. "Perceptual analysis of voice quality: the place of Vocal Profile Analysis." In William Hardcastle and Janet Beck, eds. A Figure of Speech: a Festschrift for John Laver. London/Mahwah: Lawrence Erlbaum Associates: 285-322.

Beck, Janet. 2007. Vocal profile analysis scheme: A user's manual. Edinburgh: Queen Margaret University College - QMUC, Speech Science Research Centre.

Beck, Janet. and Felix Schaeffler. 2015. "Voice quality variation in Scottish adolescents: gender versus geography”. In Maria Wolters, Judy Livingstone, Bernie Beattie, Rachel Smith, Mike MacMahon, Jane Stuart-Smith, and James M. Scobbie, eds. Proceedings of the $17^{\text {th }}$ International Congress of Phonetic Sciences. Glasgow, UK.

Devlin, Thomas, Peter French, and Carmen Llamas. 2019. "Vowel change across time, space and conversational topic: the use of localised features in former mining communities". Language Variation and Change, 31(3), 303-328.

Dickie, Catherine, Felix Schaeffler, Christoph Draxler, and Klaus Jänsch. 2009. "Speech recordings via the internet: an overview of the VOYS project in Scotland". Proceedings of Interspeech. Brighton, UK, 1807-1810.

Edmondson, Jerold A., Lama Ziwo, John H. Esling, Jimmy G. Harris, and Shaoni Li. 2001. "The aryepiglottic folds and voice quality in the $\mathrm{Yi}$ and Bai languages: laryngoscopic case studies". Mon-Khmer Studies 31: 83-100.

Esling, John H. 1978. "Voice quality in Edinburgh: a sociolinguistic and phonetic study". PhD Dissertation, University of Edinburgh. 
Foulkes, Paul. 2002. "Current trends in British sociophonetics", University of Pennsylvania Working Papers in Linguistics, 8(3).

Gold, Erica, and Peter French. 2011. "International practices in forensic speaker comparison". International Journal of Speech, Language, and the Law, 18(2): 293-307.

Gold, Erica, Sula Ross, and Kate Earnshaw. 2018. "The 'West Yorkshire Regional English Database': investigations into the generalizability of reference populations for forensic speaker comparison casework". Proceedings of Interspeech. Hyderabad, India, 27482752.

Hall-Lew, Lauren, Ruth Friskney, and James M. Scobbie. 2017. "Accommodation or political identity: Scottish members of the UK Parliament". Language Variation and Change. 29(3): 341-363.

Hillenbrand, James, Ronald A. Cleveland, and Robert L. Erickson. 1994. "Acoustic correlates of breathy vocal quality". Journal of Speech and Hearing Research, 37, 769-778.

Hirano, Minoru. 1981. Clinical examination of voice. Vienna \& New York: Springer.

Keating, Patricia, Marc Garellek, and Jody Kreiman. 2015. "Acoustic properties of different kinds of creaky voice". In Maria Wolters, Judy Livingstone, Bernie Beattie, Rachel Smith, Mike MacMahon, Jane Stuart-Smith, and James M. Scobbie, eds. Proceedings of the $17^{\text {th }}$ International Congress of Phonetic Sciences. Glasgow, UK.

Keating, Patricia, Jianjing Kuang, Christina Esposito, Marc Garellek, and Sameer Khan.2012. "Phonation in Nine Languages". Acoustical Society of America 131 (4): 3347.

Kirkham, Sam, Danielle Turton, and Adrian Leemann. 2020. "A typology of laterals in twelve English dialects." Journal of the Acoustical Society of America. 148, 1, p. EL72-EL76.

Klug, Katharina, Christin Kirchhübel, Paul Foulkes, and Peter French. 2019. "Analysing breathy voice in forensic speaker comparison: Using acoustics to confirm perception". In Sasha Calhoun, Paola Escudero, Marija Tabain and Paul Warren, eds. Proceedings of the 18th International Congress of Phonetic Sciences. Melbourne: Australasian Speech Science and Technology Association Inc., 795-799.

Kluge, Katharina, Magdalena Müller, Cornelia Dubielzig, Christoph Meinerz, and Herbert Masthoff. 2019. "Distribution of Voice Quality Features in German - Preliminary results". Paper presented at the International Association for Forensic Phonetics and Acoustics, University of Huddersfield, UK.

Knowles, Gerry O. 1978. "The nature of phonological variables in Scouse". In Peter Trudgill, ed. Sociolinguistic Patterns in British English. London: Arnold, 80-90.

Kreiman Jody, and Bruce Gerratt. 1998. "Validity of rating scale measures of voice quality". The Journal of the Acoustical Society of America 104 (3:1): 1598-608.

Ladefoged, Peter and Ian Maddieson. 1996. The Sounds of the World's Languages. Oxford: Blackwell.

Laver, John. 1980. The Phonetic Description of Voice Quality. Cambridge: Cambridge University Press.

Laver, John. 1994. Principles of Phonetics. Cambridge: Cambridge University Press.

Laver, John. 2000. "Phonetic evaluation of voice quality". In: Martin J. Ball and Kent, Raymond D., eds. Voice Quality Measurement. San Diego: Singular Publishing Group, 37-48.

Llamas, Carmen. 2007. "Place between Places": Language and Identities in a Border Town". Language in Society 36 (4): 579-604.

Moisik, Scottt, Ewa Czaykowska-Higgins, and John Esling. 2019. "Phonological potentials and the lower vocal tract". Journal of the International Phonetic Association, 1-35.

McIntyre, Dan, Lesley Jeffries, Matt Evans, Hazel Price, and Erica Gold. 2021. The Babel Lexicon of Language. Cambridge: Cambridge University Press. 
Nolan, Francis, Kirsty McDougall, Gea de Jong, and Toby Hudson. 2009. "The DyViS database: style-controlled recordings of 100 homogeneous speakers for forensic phonetic research". International Journal of Speech, Language and the Law 16(1): 3157.

San Segundo, Eugenia, Paul Foulkes, Peter French, Philip Harrison, Vincent Hughes, and Colleen Kavanagh. 2019. "The use of the Vocal Profile Analysis for speaker characterization: methodological proposals". Journal of the International Phonetic Association 49 (3): 353-380.

San Segundo Fernandez, Eugenia and Jose A. Mompean. 2017. "A Simplified Vocal Profile Analysis Protocol for the Assessment of Voice Quality and Speaker Similarity". Journal of Voice, 1-17.

Stevens, Louisa, and Peter French. 2012. "Voice quality in studio quality and telephone transmitted recordings". Paper presented at the British Association of Academic Phonetics Conference, University of Leeds, UK.

Stuart-Smith, Jane. 1999. Glasgow: "Accent and voice quality". In Paul Foulkes and Gerard J. Docherty, eds. Urban Voices: Accent Studies in the British Isles. Leeds, UK: Arnold, 201-222.

Thomas, Erik R. 2011. Sociophonetics: An Introduction. Palgrave Macmillan.

Wells, John C. 1982. Accents of English (vol. 2) Cambridge: Cambridge University Press.

Wilhelm, Stephan. 2016. "Segmental and suprasegmental change in North West Yorkshire - a new case of supralocalisation". Proceedings of the 16th Conference on Spoken English (ALOES), Villetaneuse.

Wilhelm, Stephan. 2018. "Segmental and suprasegmental change in North West Yorkshire - a new case of supralocalisation?". Corela: Cognition, representation, language, HS-24: $1-32$.

Wormald, Jessica. 2016. "Regional Variation in Panjabi-English". PhD Dissertation, University of York.

Corresponding Author:

Erica Gold

Authors's Address:

Linguistics and Modern Languages

University of Huddersfield

Queensgate, Huddersfield

HD1 3DH

Email: e.gold@hud.ac.uk 